\title{
Middle Hepatic Vein Branch-Guided Approach for Laparoscopic Resection of Liver Segment 8 Is Simple, Reliable, and Reproducible
}

\author{
Satoshi Ogiso, MD, PhD, FACS, Satoru Seo, MD, PhD, Takamichi Ishii, MD, PhD, Takayuki Anazawa, MD, PhD, \\ Kazuyuki Nagai, MD, PhD, Yoichiro Uchida, MD, PhD, Ken Fukumitsu, MD, PhD, Takashi Ito, MD, PhD, \\ Shintaro Yagi, MD, PhD, FACS, Naoko Kamo, MD, PhD, Koichiro Hata, MD, PhD, Toshihiko Masui, MD, PhD, \\ and Kojiro Taura, MD, PhD
}

Division of Hepato-Biliary-Pancreatic Surgery and Transplantation, Department of Surgery, Graduate School of Medicine, Kyoto University, Kyoto, Japan

Laparoscopic segment 8 resection is technically demanding, partly because the segment 8 Glissonean pedicle (G8) is deep in the parenchyma and difficult to identify.

\section{METHODS}

The middle hepatic vein (MHV) and its terminal branch to segment 5 (V5) are located in close proximity to the G8 origin, and recognition of V5 leads to intrahepatic identification of G8. Here, we present a video to show how the technique of V5-guided G8 identification is used in laparoscopic segment 8 resection.

\section{RESULTS}

The patient was an 81-year-old man with a solitary 35-mm hepatocellular carcinoma located deep in segment 8. A decision was made to perform laparoscopic segment 8 resection, with division of G8 at its origin. The midplane of

Electronic supplementary material The online version of this article (https://doi.org/10.1245/s10434-020-08652-x) contains supplementary material, which is available to authorized users. the liver was first transected to identify the course of MHV and V5 ramification. After dividing V5, the right anterior Glissonean pedicle was identified at the corner of the MHV and V5, followed by the isolation of G8 origin. After clamping G8, indocyanine green fluorescence staining clearly demonstrated its watershed. After dividing G8, segment 8 resection was completed with transecting the left-side, dorsal-side, and right-side borders of segment 8 .

\section{DISCUSSION}

A constant proximity of the right anterior Glissonean pedicle to the MHV and V5 has been reported. Understanding this MHV-porta hepatis relationship facilitates laparoscopic segment 8 resection. In summary, a V5-guided approach is a simple, reliable, and reproducible technique that can be safely used in laparoscopic segment 8 resection.

DISCLOSURES Satoshi Ogiso, Satoru Seo, Takamichi Ishii, Takayuki Anazawa, Kazuyuki Nagai, Yoichiro Uchida, Ken Fukumitsu, Takashi Ito, Shintaro Yagi, Naoko Kamo, Koichiro Hata, Toshihiko Masui, and Kojiro Taura have no conflicts of interest.

Publisher's Note Springer Nature remains neutral with regard to jurisdictional claims in published maps and institutional affiliations.

(C) Society of Surgical Oncology 2020

First Received: 26 February 2020;

Published Online: 27 May 2020

S. Seo, MD, PhD

e-mail: rutosa@kuhp.kyoto-u.ac.jp 